\title{
NEUTRALIZATION OF keV-IONS SCATTERED AT Cu(100)
}

\author{
J.M. VAN ZOEST, C.E. VAN DER MEIJ and J.M. FLUIT \\ Fysisch Laboratorium der Rijksuniversiteit Utrecht, Princetonplein 5, 3584 CC Utrecht, The Netherlands
}

\begin{abstract}
The scattering of $6 \mathrm{keV} \mathrm{Ne}+$ ions and dissociatively scattered $\mathrm{H}_{2} \mathrm{O}^{+}$ions (detected as $\mathrm{O}^{-}$) are measured for various exit elevation angles. Photograms show the shadowing and blocking behaviour of the scattering for different directions of ion incidence. Selected incidence directions are used to measure the yields from first and second layer reflections. The ratio of yields for $\mathrm{Cu}(100)$ shows the localized character of neutralization and electron capture. A lifetime of $(100 \pm 10)$ a.u. for $\mathrm{Ne}^{+}$against neutralization between the first two layers of a $\mathrm{Cu}(100)$ crystal was deduced.
\end{abstract}

\section{Introduction}

The strong neutralization of noble gas ions in metals is the underlying principle for the high surface sensitivity of low energy ion scattering (LEIS). For these ions the reflection region is confined to the first and second (and third) layer depending on the primary energy. The neutralization takes place in the vicinity of the surface and in the selvedge. The neutralization probability is not uniform in this region. Variation perpendicular to the surface as well as variation parallel to the surface are expected to occur, especially for $\mathrm{Cu}, \mathrm{Ag}$ and $\mathrm{Au}$ with their large number of d-electrons.

In this work low energy (6 keV) ion scattering of $\mathrm{Ne}^{+}$and $\mathrm{H}_{2} \mathrm{O}^{+}$ions by the second layer of a $\mathrm{Cu}(100)$ crystal is studied relative to the scatlering by the first layer. Large scattering angles $\left(9>45^{\circ}\right)$ are used to minimize multiple scattering effects [1] and first layer shadowing phenomena [2]. Bronckers [3] showed that for $\mathrm{Ne}^{+}$scattered from $\mathrm{Cu}(110)$ the relative $\mathrm{Ne}^{+}$intensity varied between 1 and 2 , depending on the "visibility" of the second layer. The influence of wedge focusing at this primary energy for higher elevation angles is small. The same was found (from energy dependent measurements) in our earlier work on this subject [4] for $\mathrm{Ne}^{+}$on $\mathrm{Ag}(110)$.

\section{Experiment}

The experiments are performed in the same UHV apparatus as was used in ref. [4]. The intensities of the ions can be measured for every azimuthal direction and for elevation angles of incidence between $0^{\circ}$ and $130^{\circ}$ (maximum angle between detector and ion source direction). The results of $\mathrm{Ne}^{+}$reflection for a fixed scattering angle $\vartheta=120^{\circ}$ are shown in fig. 1 : five different intensities (blackness) along the azimuthal directions give a good impression of the shadowing and blocking behaviour of the atoms in the first and second layer of the $\mathrm{Cu}(100)$ crystal. The corresponding shadowing directions can be deduced from the two layer top view of a $\mathrm{Cu}(100)$ crystal model given in fig. 2.

Note that we now use $\psi$ for the elevation angle and $\phi$ for the azimuthal angle to conform with others, different from the notation we used in ref. [4].

The $\phi=45^{\circ}, \psi=45^{\circ}$ minimum along the azimuthal direction $\langle 001\rangle$ is used as the reference signal $Y_{1}$ (for different $\vartheta$ ) where only first layer atoms can be hit. To study the neutralization behaviour of $\mathrm{Ne}^{+}$-ions between the first and second layer the yield from the first plus second layer $Y_{1+2}$ is measured (for different $\vartheta$ ) by directing the ion beam along $\psi=45^{\circ}, \phi=0^{\circ}$. The ratio $Y_{1+2} Y_{1}^{-1}-1$ is a measure of the survival probability for ions along the trajectories in the $\langle 011\rangle$ furrow.

Our scattering configuration is somewhat different from the one used by Buck et al. [5]. They used the exact $\langle 111\rangle$ axis as the incidence direction to measure $Y_{1+2}$, which mcans an elevation angle of incidence $\psi=35^{\circ}$. This is not done in our work, because we wanted to avoid different cross sections for the measurements of $Y_{1}$ and $Y_{1+2}$. The possible influence of third (fourth) layer reflections introduced by choosing this configuration is very small for $\mathrm{Ne}^{+}$scattering due to the extension of the shadow cone and to the high neutralization efficiency.

The quantity $Y_{1+2} Y_{1}^{-1}-1$ is a true measure of the relative yields of the second layer reflections in a certain range of scattering angles in this configuration. The elevation angle of incidence is $45^{\circ}$, so there is no shadowing of first layer atoms by first layer atoms. In the range of scattering angles we used, blocking occurs for $\vartheta<75^{\circ}$ as can be seen in fig. 3. This photogram is of a different type than the one shown in fig. 1 . Here the elevation angle of incidence is kept fixed. The scattering angle $\vartheta$ (and thus the ejection angle $\beta=\vartheta$ 


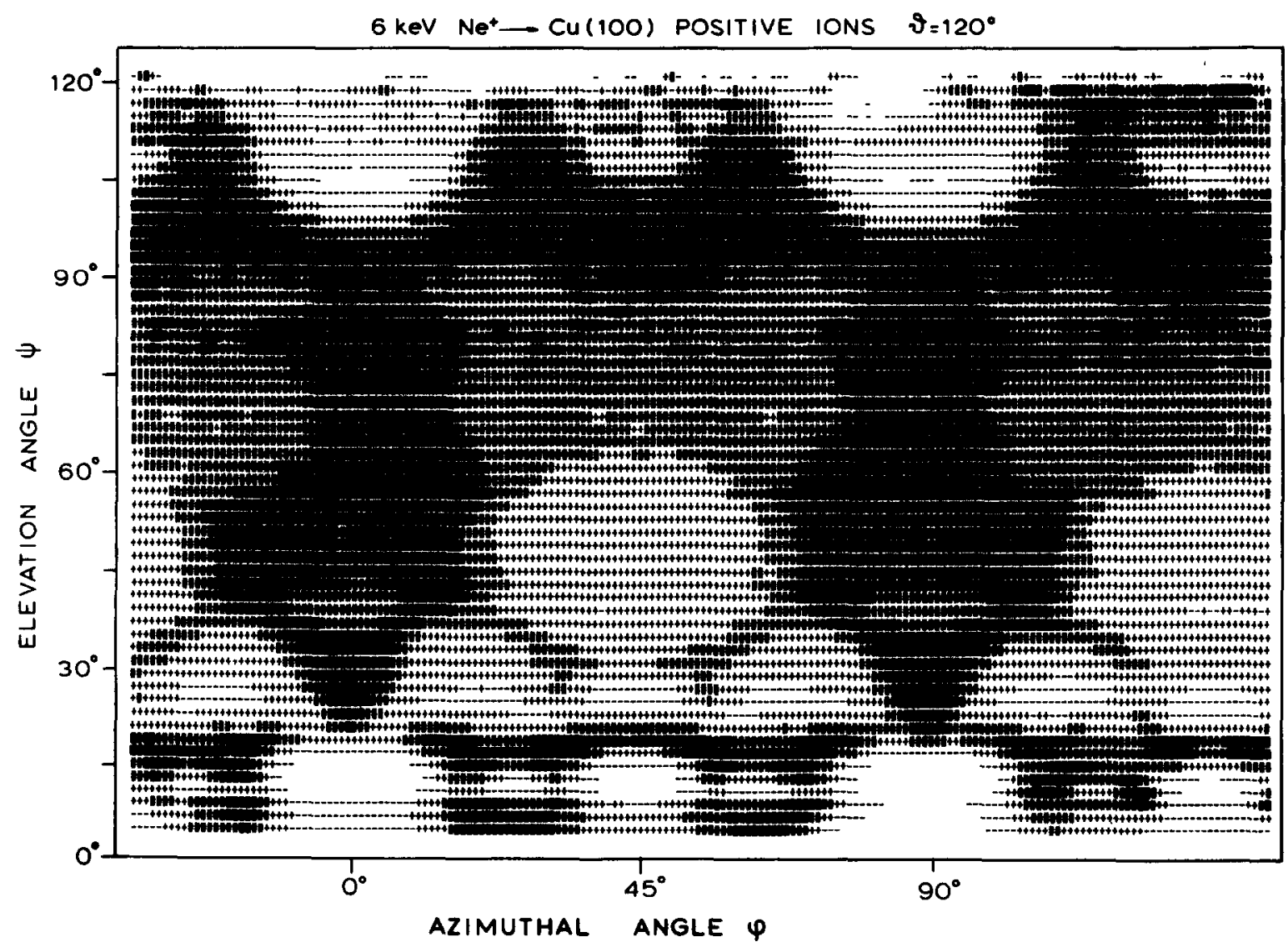

Fig. 1. Photogram of the reflected $\mathrm{Ne}^{+}$particles from a $\mathrm{Cu}(100)$ surface. The relative intensities are given in 5 grey shades for different directions of ion incidence $(\varphi, \psi)$ and a constant scattering angle $\vartheta$.
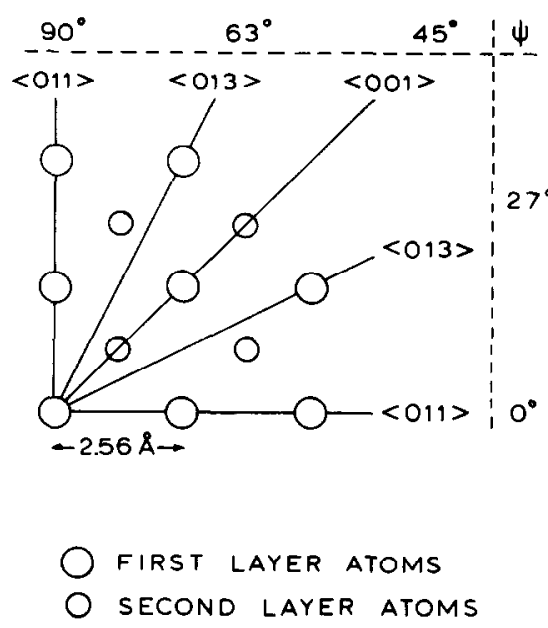

Fig. 2. Top view of the first two layers of a $\mathrm{Cu}(100)$ crystal model relevant for $\mathrm{keV}$-ion scattering: the lattice atoms can be correlated with the shadow pattern given in fig. 1. $45^{\circ}$ is varied and every two degrees an azimuthal distribution of the reflection peak is measured. From this photogram it is clear that for $\vartheta>75^{\circ}$ we are out of the blocking region. The results of these relative measurements are plotted in fig. 4 as a function of $d_{0} / v_{\perp}$, with $d_{0}=3.4$ a.u. being the distance between the first and second layer (see fig. 5) and $v_{\perp}$ the vertical velocity of the ion after scattering. The solid line in fig. 4 is calculated assuming a locally constant neutralization probability $\alpha$ per unit of time and a neutralization layer of thickness $d_{0}$. Large amounts of extra ions from the second layer at $d_{0} / v_{\perp} \approx 55$ (around $\vartheta=105^{\circ}$ ) show the insufficiency of this simple model.

\section{Localized neutralization model}

The survival probability along a certain trajectory $s$ is determined by the integral $\int[\alpha(s) / v] \mathrm{d} s$. The simple model can be improved by either allowing $\alpha$ to be locally variable, or by keeping $\alpha$ constant and allowing 


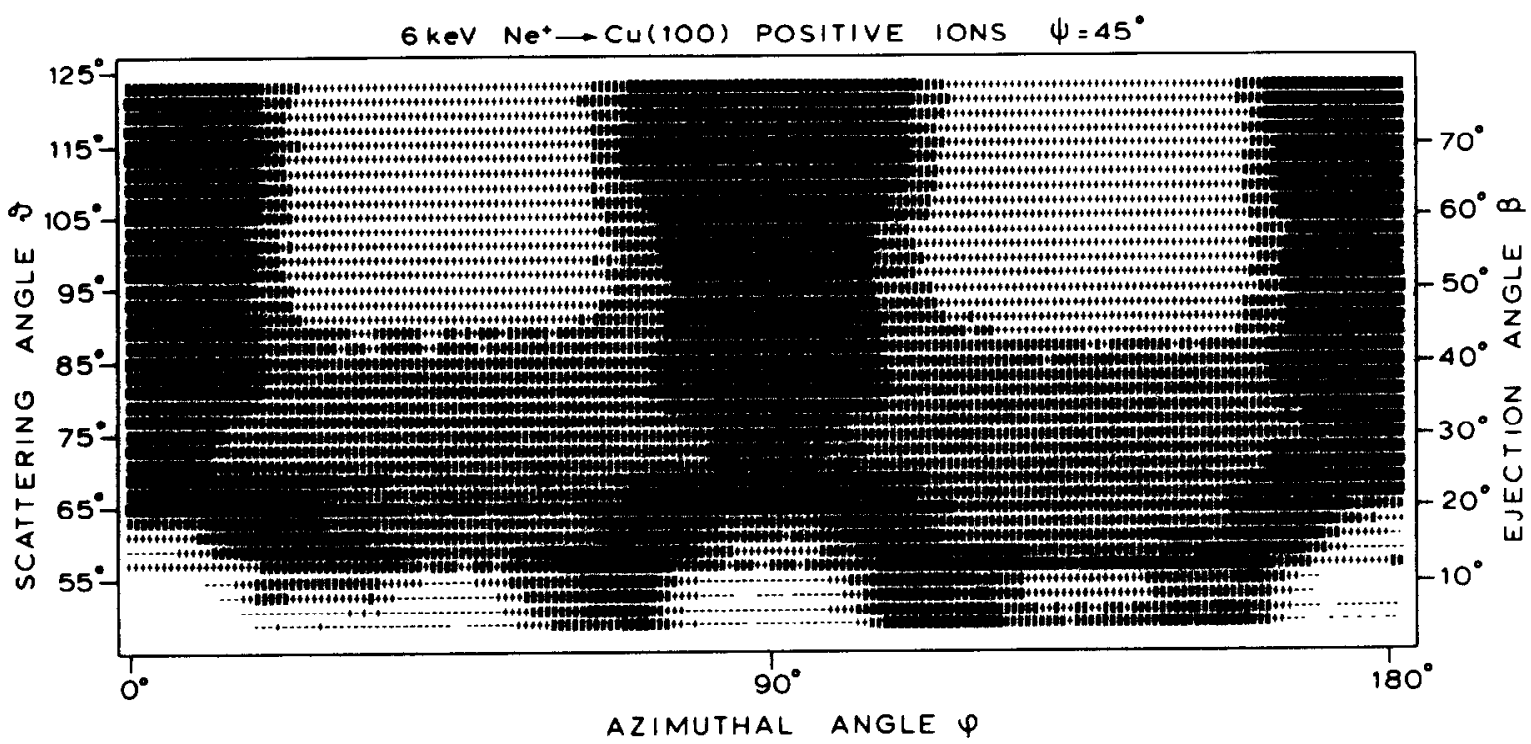

Fig. 3. Photogram of the reflected $\mathrm{Ne}^{+}$particles from a $\mathrm{Cu}(100)$ surface as a function of the different exit directions $(\varphi, \vartheta)$ for a constant elevation angle of incidence $\psi$. The ejection angle $\beta=\vartheta-45^{\circ}$.

the upper integration limit to vary according to a modulated layer thickness in stead of a constant thickness $d_{0}$. If we assume a modulated layer thickness with a modulation length given by the lattice distance $\lambda$, we are able to construct by trial and error the modulation by postulating that the survival probabilities for the various trajectories are reproduced by the model. The resulting modulated surface is shown in fig. 5 , and the agreement with the experimental data achieved with this surface is demonstrated in fig. 6, where the survival probability is plotted against

$\frac{d}{v_{\perp}}=\frac{1}{v} \int^{s(\vartheta)} \mathrm{d} s$

The improved correlation of the data points shows the adequacy of the present model (the remaining differences being within experimental uncertainties). The neutralization probability $\alpha$ resulting from the assumed

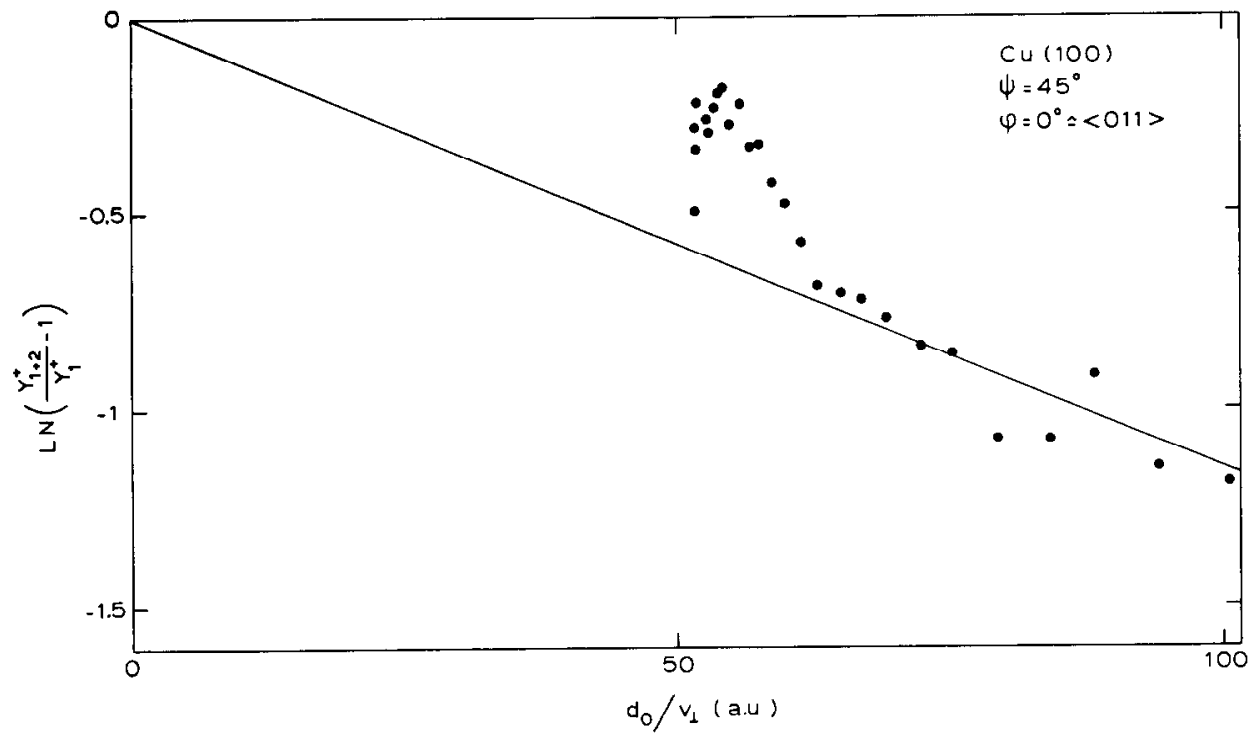

Fig. 4. The relative ion yields from second and first layer (see text) as a function of time travelled between the first and second layer of $\mathrm{Cu}(100)$. 


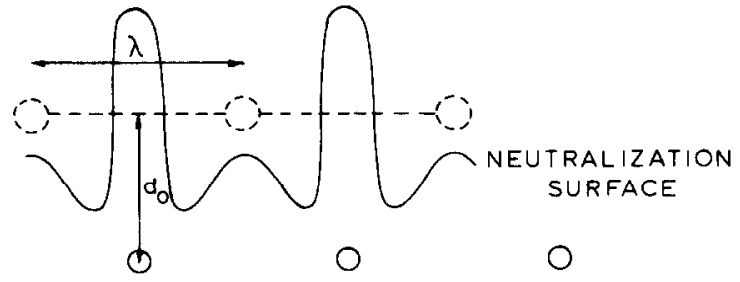

$$
\text { : FIRST LAYER ATOMS }
$$

O SECOND LAYER ATOMS

Fig. 5. A side view of two $\mathrm{Cu}(100)$ layers with the neutralization surface as deduced from the data points given in fig. 4 . The first layer atoms are out of plane.

surface shown in fig. 5 is $(0.01 \pm 10 \%)$ a.u. (see fig. 6). This corresponds to a lifetime of $(100 \pm 10)$ a.u., which is about the same as that found for Ag. This suggests that the same neutralization mechanism is operative for $\mathrm{Ne}^{+}$on $\mathrm{Cu}(100)$ and on $\mathrm{Ag}(110)$.

\section{Electron capture}

The results of relative yield measurements for negative ions formed during and after dissociative reflection of $\mathrm{H}_{2} \mathrm{O}^{+} \rightarrow \mathrm{O}^{-}$are presented in fig. 7. Two values for one scattering angle gives an indication of the accuracy.

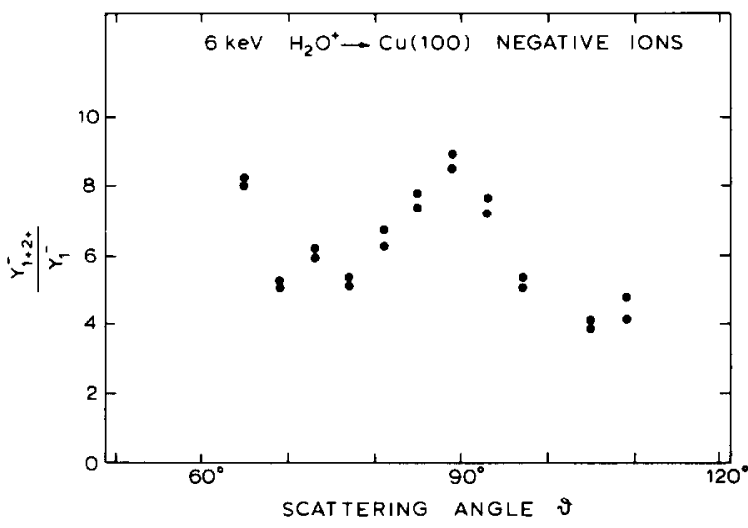

Fig. 7. Relative $\mathrm{O}^{-}$yields from $\mathrm{H}_{2} \mathrm{O}^{+}$ions predominantly scattered from second and first atomic layers of a $\mathrm{Cu}(100)$ crystal.

The formation of negative ions is enhanced upon penetration and relative yields of 3 to 9 are found. In this situation third and fourth layer reflections may be possible for those directions where no blocking on the outgoing trajectory is expected (blocking at $\beta=\vartheta-45^{\circ}$ values of $35^{\circ}, 55^{\circ}$ and $90^{\circ}$ ). A pronounced minimum is found for $\vartheta=105^{\circ}$. This is related to the maximum found for the surviving positive projectiles (fig. 4).

\section{Conclusions}

The localized character of neutralization of $\mathrm{Ne}^{+}$ between the first and second layer in $\mathrm{Cu}(100)$ is ap-

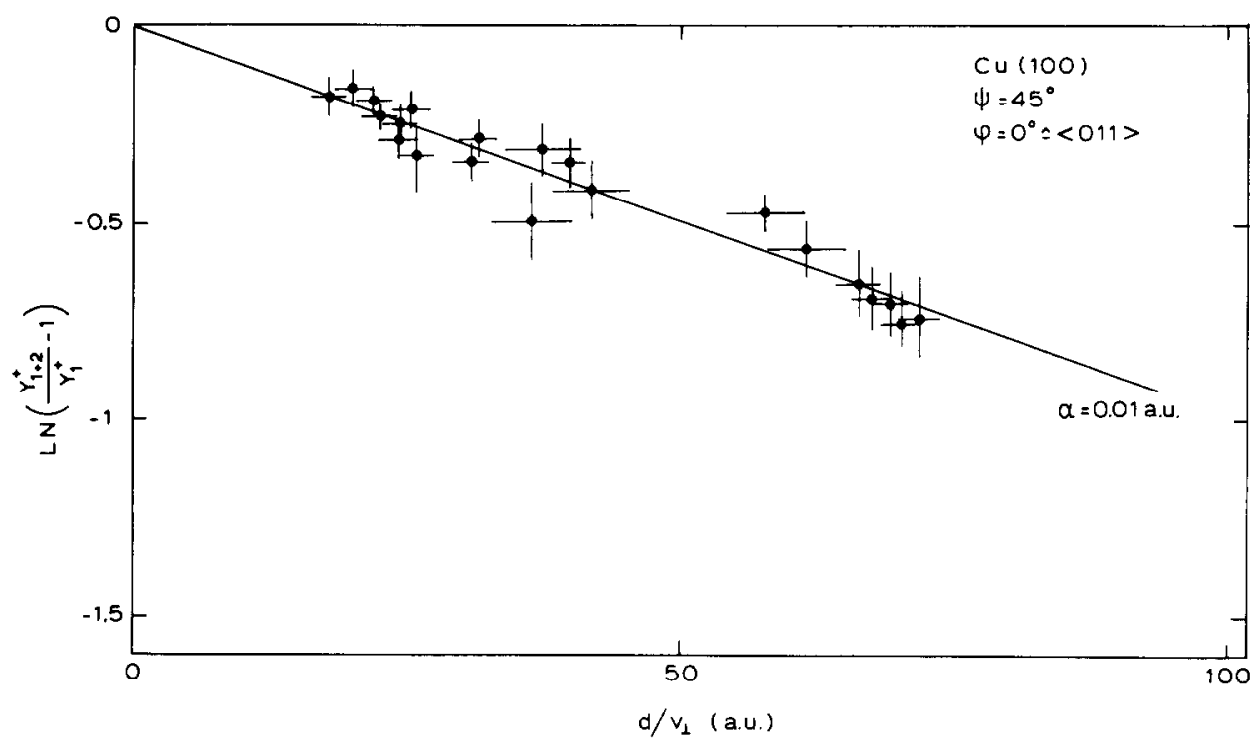

Fig. 6. The relative ion yields from the second and first layers as a function of the time travelled between the second layer and the neutralization plane. $d$ is the normal projection of the path of the projectile on the outward trajectory between the second layer and the neutralization surface (all parts of the path below the surface are counted). 
parent. The neutralization mcchanism operative in $\mathrm{Cu}$ and $\mathrm{Ag}$ is the same. The qualitative difference between $\mathrm{Cu}(100)$ and $\mathrm{Ag}(100)$ suggests that the main difference is caused by the surface structure and not by the kind of the metal. Although this method is somewhat arbitrary we believe it gives a fair idea of the possible electron density variation in the first surface layer.

We thank Professor A. Niehaus for the stimulating cooperation during the work described in this paper.

This work was performed as part of the research programme of the "Stichting voor Fundamenteel Onderzoek der Materie" (FOM) with financial support from the Nederlandse Organisatie voor Zuiver Wetenschappelijk Onderzoek" (ZWO).

\section{References}

[1] L.K. Verhey, B. Poelsema and A.L. Boers, Rad. Effects 31 (1976) 23.

[2] R.P.N. Bronckers and A.G.J. de Wit, Surface Sci. 104 (1981) 384.

[3] R.P.N. Bronckers and A.G.J. de Wit, Surface Sci. 112 (1981) 111.

[4] A.G.J. de Wit, J.M. van Zoest and J.M. Fluit, Surface Sci. 126 (1983) 589.

[5] T.M. Buck, G.H. Wheatley and L.K. Verhey, Surface Sci. 90 (1979) 635. 